\title{
Novel Rapid Culture-Based Detection Method for Methicillin-Resistant Streptococcus aureus
}

In the May 2008 issue of Journal of Clinical Microbiology, Malhotra-Kumar et al. (2) provided an excellent minireview of current trends in rapid diagnostics for methicillin-resistant Staphylococcus aureus (MRSA) and glycopeptide-resistant enterococci. In this article, the authors describe a novel rapid culture-based assay, the 3M BacLite rapid MRSA test, which detects MRSA without the need for macroscopic growth of bacterial colonies, allowing the presence or absence of MRSA to be determined within $5 \mathrm{~h}$. The article states the assay detects ciprofloxacin-resistant MRSA and comments that this may lead to the possibility of the assay not detecting communityacquired MRSA strains, as well as some hospital-acquired MRSA strains, that are sensitive to ciprofloxacin. The authors are correct in their statements but refer to an assay formulation that has not been available since February 2007, when ciprofloxacin was replaced by cefoxitin as a selective agent.

The ability of the $3 \mathrm{M}$ BacLite rapid MRSA test to detect MRSA strains from a diverse genetic background has been evaluated by von Eiff et al. (4). In an extensive study, more than 700 methicillin-susceptible and methicillin-resistant strains, covering $>90 \%$ of all registered European MRSA spa types within the SeqNet network, were tested using the 3M BacLite rapid MRSA test. The study reported that all 513 MRSA strains tested were correctly recognized as methicillin resistant while none of the 211 methicillin-susceptible strains were detected as positive. In an additional study (5), 27 MRSA strains recovered from pigs, which have been reported to cause detection problems in some molecular assays, were correctly detected as positive by the $3 \mathrm{M}$ BacLite rapid MRSA test. In addition to these analytical studies, there are an increasing number of studies assessing the performance of this assay with clinical specimens. Our group (3) reported a diagnostic sensitivity of $94.6 \%$ and a diagnostic specificity of $96.9 \%$ for nasal screening swabs and $95.9 \%$ sensitivity and $88.8 \%$ specificity for groin screening swabs when the assay was referenced to standard operating procedures at three hospital sites in the United Kingdom. More recently Cohen et al. (1) assessed the performance characteristics of the 3M BacLite rapid MRSA assay for a range of body sites compared to chromagar and a commercial molecular assay and concluded that the BacLite assay's performance, ease of use, and capacity best met the needs of the authors' hospital for rapid MRSA screening.

The limitations of traditional diagnostic methods coupled with the increase in antimicrobial resistance means that there is a growing demand among health care providers for rapid microbiological tests to assist patient care and disease control. Malhotra-Kumar et al.'s excellent minireview demonstrates the range of options available to clinical microbiologists today and the fast-moving nature of this field.

\section{REFERENCES}

1. Cohen, D., S. OHara, B. Bagoole, and M. Almeida. 2008. Evaluation of the 3M BacLite rapid MRSA test for the direct detection of MRSA from nasal, groin, and combined nasal/axilla/groin surveillance specimens, abstr. P2132. Abstr. 18th Eur. Congr. Clin. Microbiol. Infect. Dis. European Society of Clinical Microbiology and Infectious Diseases, Basel, Switzerland.

2. Malhotra-Kumar, C., K. Haccuria, M. Michiels, M. Ieven, C. Poyart, W. Hryniewicz, and H. Goossens. 2008. Current trends in rapid diagnostics for methicillin-resistant Staphylococcus aureus and glycopeptide-resistant Enterococcus species. J. Clin. Microbiol. 46:1577-1587.
3. OHara S., S. Gregory, D. Taylor, A. Gough, and N. Foote. 2007. Evaluation of the 3M BacLite rapid MRSA test for the direct detection of MRSA from nasal and groin surveillance specimens, abstr. 540, p. 162-163. Abstr. Annu. Meet. Infect. Dis. Soc. Am. Infectious Diseases Society of America, Arlington, VA.

4. von Eiff, C., D. Maas, G. Sander, A. W. Friedrich, G. Peters, and K. Becker. 2008. Microbiological evaluation of a new growth-based approach for rapid detection of methicillin-resistant Staphylococcus aureus. J. Antimicrob. Chemother. 61:1277-1280.

5. von Eiff, C., D. Maas, G. Sander, A. Eggemann, A. W. Friedrich, G. Peters, and K. Becker. 2008. Microbiological evaluation of a new growth-based approach designated for rapid detection of methicillin-resistant Staphylococcus aureus directly from specimens, abstr. P1760. Abstr. 18th Eur. Congr. Clin. Microbiol. Infect. Dis. European Society of Clinical Microbiology and Infectious Diseases, Basel, Switzerland.

\author{
Stephen OHara \\ $3 M$ Medical Diagnostics \\ 1 Morley Street \\ Loughborough, Leicestershire LE11 1EP \\ United Kingdom
}

Phone: 0044(0)7887874392

E-mail: sohara@mmm.com

\section{Authors' Reply}

We thank Dr. OHara for bringing to attention this important information not available at the time of our initial publication (3). While the previous version of the 3M BacLite rapid MRSA test would fail to detect community-acquired methicillin-resistant Staphylococcus aureus (MRSA) and ciprofloxacin-sensitive hospital-acquired MRSA (3), the new 3M BacLite assay correctly differentiates MRSA and methicillin-sensitive $S$. aureus (MSSA) strains and detects MRSA from screening samples, as reported recently $(1,4)$. We also validated the new $3 \mathrm{M}$ BacLite assay with a limited number of well-characterized strains $(n=52)$ comprising 24 hospital- or community-acquired or animal MRSA strains carrying SCCmec types I to V or nontypeable $S C C m e c$ cassettes; 5 MSSA strains; 5 methicillin-resistant, coagulase-negative staphylococci (MRCoNS); 3 methicillin-sensitive, coagulase-negative staphylococci (MSCoNS); and 15 strains of other commonly encountered bacteria (Escherichia coli, Enterococcus spp., Citrobacter freundii, Pseudomonas aeruginosa, Enterobacter cloacae, and Klebsiella spp.). The BacLite assay correctly identified all 24 MRSA strains from highly diverse molecular backgrounds, and none of the MSSA or MSCoNS strains were reported positive by the test. However, of the five MRCoNS strains, four were identified as MRSA. Also, among the 15 strains of other bacteria that we tested, $C$. freundii and Enterococcus faecalis (1 strain each) gave false-positive results. Thus, the diagnostic sensitivity and specificity of the new 3M BacLite assay were $100 \%$ and $79 \%$, respectively, in our hands with this limited number of samples. Cross-reactivity with MRCoNS has also been noted by the manufacturers, possibly because of nonspecific binding of CoNS during the immunomagnetic separation step. Nonetheless, the BacLite assay cleanly sidesteps the problems faced by DNA sequence-based detection methods due to the tremendous sequence variations observed in the SCCmec cassette (2), resulting in an impressive sensitivity that should withstand 
the constant emergence of novel SCCmec variants. In conclusion, the new 3M BacLite assay is a promising assay that combines the advantages of culture with the rapidity of molecular method-based detection.

\section{REFERENCES}

1. Cohen, D., S. OHara, B. Bagoole, and M. Almeida. 2008. Evaluation of the 3M BacLite rapid MRSA test for the direct detection of MRSA from nasal, groin, and combined nasal/axilla/groin surveillance specimens, abstr. P2132. 18th Eur. Congr. Clin. Microbiol. Infect. Dis. European Society of Clinical Microbiology and Infectious Diseases, Basel, Switzerland.

2. Francois, P., M. Bento, G. Renzi, S. Harbarth, D. Pittet, and J. Schrenzel. 2007. Evaluation of three molecular assays for rapid identification of methicillin-resistant Staphylococcus aureus. J. Clin. Microbiol. 45:20112013

3. Malhotra-Kumar, S., K. Haccuria, M. Michiels, M. Ieven, C. Poyart, W. Hryniewicz, and H. Goossens. 2008. Current trends in rapid diagnostics for methicillin-resistant Staphylococcus aureus and glycopeptide-resistant Enterococcus species. J. Clin. Microbiol. 46:1577-1587.
4. von Eiff, C., D. Maas, G. Sander, A. W. Friedrich, G. Peters, and K. Becker. 2008. Microbiological evaluation of a new growth-based approach for rapid detection of methicillin-resistant Staphylococcus aureus. J. Antimicrob. Chemother. 61:1277-1280.

\author{
Surbhi Malhotra-Kumar*† \\ Kelly Haccuria \\ Liesbet Van Heirstraeten \\ Margareta Ieven \\ Herman Goossens \\ Department of Medical Microbiology \\ Vaccine and Infectious Disease Institute \\ Universiteit Antwerpen \\ Antwerp, Belgium \\ *Phone: 32-3-820-25-51 \\ Fax: 32-3-820-26-63 \\ E-mail: surbhi.malhotra@ua.ac.be
}

$\dagger$ All authors are writing on behalf of the MOSAR WP2 Study Team. 